\title{
Estrategias de intervención para mejorar la calidad de vida en pacientes con insuficiencia cardíaca
}

Marcela González ${ }^{1}$, Víctor Pedrero².

\author{
1 Enfermera Matrona \\ 2 Instructor \\ Escuela de Enfermería, Pontificia Universidad Católica de Chile.
}

Objetivos: Conocer las características de las estrategias de intervención multidisciplinarias implementadas por el equipo de salud que mejoran la calidad de vida de los pacientes con insuficiencia cardíaca.

Métodos: Se consultó las bases de datos Pubmed, Cochrane library, Proquest y CINHAL entre mayo y junio del 2011, encontrándose 99 artículos de los cuales se seleccionaron 40 (estudios randomizados controlados, metanálisis y revisiones sistemáticas) publicados en los últimos 10 años.

Resultados: Entre las variables que tienen mayor efecto sobre la calidad de vida de los pacientes con insuficiencia cardíaca se encuentran: intervenciones educativas (reconocimiento de signos y síntomas, adherencia al tratamiento farmacológico, cambios del estilo de vida, peso diario, etc.); consejería telefónica o video llamadas (ajuste de terapia diurética); y actividad física (prueba de caminata de 6 minutos, ejercicios aeróbicos, etc.). La implementación de estrategias de intervención multidisciplinarias tiene un efecto positivo en el incremento de la calidad de vida. Existe una relación significativa entre las sub-escalas de calidad de vida (funcionamiento físico, salud general y mental), y del puntaje total de la dimensión física con el riesgo de readmisión hospitalaria.

Conclusión: Intervenciones multidisciplinarias de tipo educativo y aquellas orientadas a mejorar la condición física tienen efecto positivo en mejorar la calidad de vida de los pacientes con Insuficiencia Cardíaca. Ello se relaciona con una disminución del riesgo de re-hospitalización.

Palabras claves: Insuficiencia Cardíaca; calidad de vida; educación para la salud; autocuidado en salud. 


\section{Interventional strategies to improve quality of life in patients with Heart Failure.}

Aim: to analyze interventional strategies that have been used to improve quality of life in patients with heart failure

Methods: through a review of the Pubmed, Cochrane Library, Proquest and CiNHAL data bases 99 papers published in the last 10 years were found. Among them, 40 randomized control studies, metaanalysis or systematic reviews were selected for the analysis

Results: education (symptoms and signs recognition, adherence to drug treatments, life style changes, daily wieghing, etc), phone or video calls (adjustment of diuretic therapy) and activities designed to improve physical condition were the most effective interventions. A multi-disciplinary approach using these interventions has a positive effect on subscales of quality of life (physical function, general and mental health) as well as upon the physical dimension overall score. In turn, these effects are related to a decreased risk of re-admision for heart failure.

Conclusion: Educational interventions along with aimed at improving the physical condition effectively improve the quality of life and reduce de risk of re-admission in patients with heart failure.

Keywords: Heart Failure; quality of life; health education; selfcare.

\section{Introducción}

La Insuficiencia cardíaca (IC) es un síndrome de proporciones epidémicas que actualmente presenta una prevalencia general en USA que se estima entre 5 y 6 millones de habitantes, con una incidencia anual de 10 por cada 1.000 personas después de los 65 años ${ }^{1,2,3}$. Se estima un incremento de los casos de IC en un $24 \%^{3}$ para el año 2020; junto con esto la mortalidad general en personas adultas con IC es aproximadamente $40 \%$; la tasa anual para todas las causas de readmisión hospitalaria de estos pacientes es $64 \%$, lo que consume alrededor del $2 \%$ del gasto en salud de los países desarrollados ${ }^{4}$.

En los últimos años se han implementado diferentes estrategias tendientes a mejorar el pronóstico y disminuir las readmisiones hospitalarias, entre ellas programas de seguimiento, educación y consultoría con diversos profesionales (enfermera(o), nutricionista, kinesiólogo y médico). Un meta-análisis mostró que las intervenciones multidisciplinarias reducían en un 3\% la mortalidad y un $8 \%$ las readmisiones hospitalarias ${ }^{5}$.

La cronicidad y pronóstico de la IC han provocado interés en ampliar la perspectiva con que está siendo abordado su manejo $y$, por tanto, se ha considerado la calidad de vida como un aspecto que podría otorgar orientación

al momento de elegir las estrategias para el manejo de los pacientes con un mayor impacto en la evolución natural de la enfermedad ${ }^{6,7}$. Al rededor del mundo, diversas experiencias de intervención única o múltiple han tenido éxito en mejorar la calidad de vida de personas con $\mathrm{IC}^{8,9}$.

El objetivo de esta revisión bibliográfica es conocer las características de las estrategias de intervención multidisciplinarias implementadas por el equipo de salud, que han mejorado la calidad de vida de los pacientes con insuficiencia cardíaca.

\section{Método}

Para la siguiente revisión de la literatura se han consultado las bases de datos Pubmed, Cochrane library, Proquest, Scielo y CINHAL. Estas bases de datos fueron seleccionadas porque son los recursos más completos para la enfermería, psicológicos y de salud biomédica. Para la búsqueda inicial de artículos se utilizó una combinación de las palabras claves "heart failure", "disease management programs", "health related quality of life". Los criterios de inclusión para la selección de artículos fueron los siguientes: (a) artículos escritos en inglés o español, (b) publicados en los últimos 10 años, y (c) focalizados en calidad de vida, programas de intervención e IC. Se excluyeron 
estudios en pacientes pediátricos.

La búsqueda inicial dio como resultado 99 artículos. Después de leer los resúmenes de cada uno de ellos, 40 artículos fueron seleccionados para esta revisión bibliográfica por tratarse de estudios randomizados controlados, metaanálisis o revisiones sistemáticas.

\section{Resultados}

\section{Calidad de Vida e Insuficiencia Cardíaca}

A pesar de que en los últimos años se han producido numerosos avances en el tratamiento de los pacientes con IC, ésta ha permanecido como una causa importante de morbimortalidad en el mundo ${ }^{3,4}$. Considerando la cronicidad y el pronóstico de esta enfermedad, se ha visto un interés progresivo en el estudio de la calidad de vida ${ }^{6}$.

La calidad de vida ha sido medida a través de diferentes instrumentos, tanto genéricos (Medical Outcomes Study - Item Short Form SF36, Nottingham Health Profile), como específicos (Minessota Living Heart Failure MLHF, Kansas City Cardiomiophaty) $6,7,10,11$.

Se ha establecido que los puntajes de los diferentes instrumentos empeoran en relación a la progresión de la enfermedad $^{6}$ En la literatura se encuentran diferentes factores que condicionan estos resultados ${ }^{7}$. Por ejemplo, Gott et al., en el año 2006, realizaron un ensayo clínico en el cual identificaron como factores predictivos de empeoramiento de la calidad de vida: el sexo femenino, la clasificación funcional de NYHA III o IV, la evidencia de depresión, el pertenecer a grupos socioeconómicos vulnerables y tener dos o más co-morbilidades; además, establecieron que la edad y la calidad de vida se relacionaban de forma inversa en el grupo de pacientes estudiados ${ }^{12}$.

Por otra parte, ha sido ampliamente aceptada la relación directa entre la calidad de vida y capacidad funcional; sin embargo, algunas investigaciones afirman que solo existe una relación moderada de la capacidad funcional con la calidad de vida de los pacientes con IC ${ }^{13,14}$.

Existen escasos estudios que relacionen calidad de vida con la etapificación actual de la IC establecida en las guías clínicas del American College of Cardiology y American Heart Association (ACC/AHA). Sin embargo, Azevedo et al., afirmaron que existe una relación directa de esta etapificación con el deterioro de la calidad de vida, medida a través del instrumento SF36. El grupo en estadio $\mathrm{C}$ presenta significativamente peores valores en todos los dominios salvo en salud mental, comparados con los pacientes de bajo riesgo. Por otra parte, en la sub-escala de funcionamiento social también el grupo en estadio B presentó una diferencia significativa, al relacionarlo con el grupo de bajo riesgo ${ }^{6}$.

\section{Estrategias de Intervención}

Recientemente ha cobrado importancia el estudio de intervenciones no farmacológicas que permitan mejorar el pronóstico de estos pacientes Se ha demostrado que estas intervenciones tienen el potencial de reducir la mortalidad y rehospitalizacion, mejorar la calidad de vida y ser económicamente atractivos; sin embargo, la reducción de riesgo en los diferentes estudios varía ampliamente lo que puede deberse a la heterogeinicidad de las estrategias ${ }^{5,15,16,17}$. De manera general, estos programas se enfocan, principalmente en la educación en salud y el apoyo continuo del paciente después del alta hospitalaria. Se han descrito los tipos de intervención que tendrían mayor efecto sobre la calidad de vida de los pacientes con IC y entre ellas se encuentran: intervenciones educativas (reconocimiento de signos y síntomas, adherencia al tratamiento farmacológico, cambios del estilo de vida, peso diario, diario de seguimiento, charlas, etc.; consejería telefónica o video llamadas (ajuste de terapia diurética); y actividad física (prueba de caminata de 6 minutos, ejercicios aeróbicos, juegos con balón, etc.), entre otras ${ }^{11}$.

\section{Intervenciones Educativas}

En la mayoría de los estudios se ha detectado que con intervenciones educativas, seguidas a 6 y 12 meses, se obtiene una mejoría en el puntaje total de los instrumentos de medición de calidad de vida (SF36, MLWHF, Kansas City Cardiomiophaty questionnaire) $)^{18,19,20,21}$.

$\mathrm{Al}$ realizar un análisis diferenciado del impacto de las estrategias que incluyen actividades educativas, con las diferentes dimensiones medidas, la mejoría de la calidad de vida está asociada a las dimensiones física y emocional $18,19,22,23,24$.

Un ejemplo del diseño de este tipo de estrategia es la descrita por Doughty et al., quienes aplicaron el MWLHF en una población de pacientes con IC en capacidad funcional III a IV de la NYHA con una fracción de eyección de aproximadamente $30 \%$, que fueron sometidos a una intervención médica apoyada con una estrategia educativa rea- 
lizada por un profesional de enfermería, obteniendo una mejoría en la dimensión física a los 12 meses respecto de los controles $(\mathrm{p}=0,015)^{8}$. Esta estrategia constaba de una visita al paciente antes de su alta donde se le entregaba un diario en el cual debía registrar peso diario, su lista de medicamentos y anotaciones de las sesiones educativas grupales. Estas sesiones se realizaron con la participación del cardiólogo y la enfermera del estudio, por 1 y media a 2 hrs. En ellas se abordó: explicación de los signos y síntomas de la IC, importancia del monitoreo diario del peso $\mathrm{y}$ las acciones planeadas en torno a los cambios, efecto de los medicamentos, la importancia de la adherencia a ellos y recomendaciones en torno a la dieta y el ejecicio ${ }^{8}$. En un estudio randomizado controlado Wierzchowiecki et al. utilizando una estrategia educativa similar, en el contexto de un programa multidisciplinario, obtuvieron una mejoría de la calidad de vida (MWLHF) a 12 meses $(\mathrm{p}<0.001)^{25}$.

En el estudio REMADHE, la mejoría de los puntajes de calidad de vida a 5 años se mantuvo siempre mejor que en el grupo control ${ }^{26}$.

Delaney y Apostolidis utilizando intervenciones educativas realizadas a domicilio por enfermeras mostraron una mejoría significativa de la calidad de vida de pacientes con IC en relación al grupo control $(\mathrm{p}<0.01)^{27}$. También se ha detectado una mejoría en la calidad de vida en pacientes en quienes se han realizado intervenciones combinadas con la visita domiciliaria ${ }^{9,25}$. Iguales beneficios en comparación al grupo control se han demostrado con intervenciones educativas efectuados por 12 semanas post alta ${ }^{16}$.

\section{Consejería telefónica o video llamadas}

Kasper et al, realizando consejería telefónica para identificar problemas y aconsejar medidas a seguir según un algoritmo determinado, observaron menor sintomatología en pacientes con IC comparados a controles $(\mathrm{p}=0.003)$. Tanto el puntaje total, como en las dimensiones física y emocional del MWLHF revelaron una mejoría en la calidad de vida en el grupo intervenido 9 .

Otro estudio realizado en Argentina recientemente, en donde se efectuó una intervención telefónica similar, mostró que los pacientes del grupo intervención presentaron mejores puntajes de calidad de vida en el cuestionario de MWLHF, tanto en el puntaje global ( $p=0,001)$, como en los dominios físicos $(\mathrm{p}=0,007)$ y emocionales $(\mathrm{p}=$
$0,002)^{29}$. Un meta-análisis corroboró que el monitoreo telefónico mejoraba la calidad de vida en los pacientes con IC congestiva. La estrategia usada en la mayoría de los estudios consiste en intervención efectuada por enfermería, obteniendo información de síntomas y signos, efectuando apoyo educativo y aconsejando ajustes de tratamiento farmacológico. En algunos casos se ha usado videoconferencia telefónica ${ }^{30}$.

\section{Actividad Física}

Estrategias multidisciplinarias han incluido también el ejercicio como parte de la rehabilitación de los pacientes con IC. En la mayoría de los casos, éste es guiado por un fisioterapista o kinesiólogo y también por profesionales de enfermería $5,25,31$.

Gary et al., utilizaron la caminata 3 veces por semana durante 12 semanas, monitorizada por tolerancia, síntomas y signos (disnea, fatiga, frecuencia cardíaca). Los pacientes debían incrementar el tiempo de ejercicio 5 min de acuerdo a la tolerancia con un máximo de 1 hora. Compararon la efectividad del ejercicio y la terapia educativa, aislados o en combinación, demostrando un mejor efecto de la última $^{32}$. Otros estudios han también apoyado el beneficio obtenido por el entrenamiento físico sobre la calidad de vida en pacientes con $\mathrm{IC}^{33,34}$.

Un meta-análisis realizado en el año 2006, concluyó que existía un mejoramiento significativo de la calidad de vida con una diferencia de 9,7 puntos en el puntaje general, en aquellos pacientes que eran sometidos a la prueba de caminata de 6 minutos, ejercicios aeróbicos, ejercicios de calentamiento muscular y juegos de balón. ${ }^{35,36}$. Hwang et al., confirmaron estos hallazgos, reportando que el ejercicio mejoraba significativamente la calidad de vida ${ }^{37}$.

\section{Impacto sobre re-hospitalizaciones y mortalidad}

Rodríguez-Artalejo et al., encontraron que existía una relación significativa entre las sub-escalas de funcionamiento físico, salud general, salud mental y el puntaje total de la dimensión física del SF36, y el riesgo de readmisión hospitalaria. Por otro lado el riesgo relativo de morir es más del doble cuando los puntajes del funcionamiento físico, vitalidad, salud mental y el puntaje sumativo del score emocional empeoran ${ }^{38}$. Se ha mostrado que puntajes deficientes en los dominios físicos y emocionales son predictores independientes de mortalidad a largo plazo ${ }^{38}$. 
Un efecto similar se observa al aplicar el MLWHF ${ }^{7}$.

\section{Discusión}

La implementación de estrategias de intervención multidisciplinarias y de seguimiento riguroso de los pacientes con IC, podría generar un cambio en el comportamiento epidemiológico de esta población, una mejora en la calidad de los años de sobrevida y, a largo plazo, una disminución de los gastos en salud de los países. Cabe destacar que en Europa el costo por hospitalizaciones donde la IC fue la primera causa de admisión aumentó de 1,3 a 2,1\% entre el 1990 y el 2000, llegando a un $4 \%$ cuando no consideramos a la IC como primera causa de ingreso ${ }^{39}$. El costo efectividad de los programas de intervención ha sido menos estudiado, pero hay alguna evidencia de que si pueden ser costo-efectivos ${ }^{9,31,40}$.

La evidencia presentada es categórica en demostrar que la intervención educativa es el eje de la elaboración de cualquier programa que busque tener algún impacto sobre la calidad de vida de las personas con insuficiencia cardíaca. Así mismo, es interesante destacar que en la mayoría de los estudios se considera la presencia del médico y personal de enfermería como básicos para implementar los programas. Sin embargo, es recomendable que el abordaje del paciente sea efectivamente multidisciplinario integrando la visión de profesionales del área de la kinesiología, nutrición y sicología.
Existe evidencia que una estrategia multidisciplinaria y holística como la efectuada por Capomolla et al., es capaz de reducir cerca de un $70 \%$ las readmisiones hospitalarias ( $86 \%$ de readmisiones en el grupo control versus $14 \%$ en el grupo intervención $(\mathrm{p}<0,00001)$.

Actualmente, no existe en Chile una política orientada a la implementación de los programas mencionados, política que debiera implementarse, dada la fuerte evidencia presentada en esta revisión. Por otra parte, aun cuando se han conocido los dominios más beneficiados con las intervenciones multidiscipinarias, es un desafío la evaluación del impacto de estas estrategias en otros ámbitos de la vida, no abordados por los instrumentos de calidad de vida (vida sexual, familiar, autoimagen, etc.)

En conclusión, la implementación de intervenciones educativas presenciales o a distancia y multidisplinarias puede generar profundos cambios en el pronóstico y la calidad de vida de los pacientes con insuficiencia cardíaca. Ello implica un gran desafío que involucra voluntad política y esfuerzo del equipo de salud, como también, y en forma muy importante, del paciente.

\section{Agradecimientos}

Los autores agradecen a Alejandra Araya Gutiérrez y María Teresa Urrutia, por su valioso apoyo en análisis y redacción de los datos.

\section{Referencias:}

1. THOM T, HAASE N, ROSAMOND W, HOWARD VJ, RUMSFELD J, MANOLIO T, et al. Heart Disease and Stroke Statistics--2006 Update: A Report From the American Heart Association Statistics Committee and Stroke Statistics Subcommittee. Circulation. 2006;113:e85-151.

2. HODGES P. Heart Failure: Epidemiologic Update. Crit Care Nurs Q. 2009;32:24-32.

3. MOSTERD A, HOES AW. Clinical epidemiology of heart failure. Heart. 2007;93:1137 -1146

4. CURTIS LH, GREINER MA, HAMMILL BG, DIMAR-
TINO LD, SHEA AM, HERNANDEZ AF, et al. Representativeness of a National Heart Failure Quality-of-Care Registry: Comparison of OPTIMIZE-HF and Non-OPTIMIZE-HF Medicare Patients. Circ Cardiovasc Qual Outcomes. 2009;2:377-384.

5. GÖHLER A, JANUZZI JL, WORRELL SS, OSTERZIEL KJ, SCOTT GAZELLE G, DIETZ R, et al. A Systematic Meta-Analysis of the Efficacy and Heterogeneity of Disease Management Programs in Congestive Heart Failure. Journal of Cardiac Failure. 2006;12:554-567.

6. AZEVEDO A, BETTENCOURT P, ALVELOS M, MARTINS E, ABREU-LIMA C, HENSE H, et al. Health-related quality of life and stages of heart failure. International Journal of Cardiology. 2008 Sep 26;129:238-244. 
7. JOHANSSON P, AGNEBRINK M, DAHLSTRÖM U, BROSTRÖM A. Measurement of health-related quality of life in chronic heart failure, from a nursing perspective--a review of the literature. European Journal of Cardiovascular Nursing. 2004;3:7-20.

8. DOUGHTY R, WRIGHT S, PEARL A, WALSH H, MUNCASTER S, WHALLEY G, et al. Randomized, controlled trial of integrated heart failure management. The Auckland Heart Failure Management Study. European Heart Journal. 2002;23:139-146.

9. KASPER EK, GERSTENBLITH G, HEFTER G, VAN ANDEN E, BRINKER JA, THIEMANN DR, et al. A randomized trial of the efficacy of multidisciplinary care in heart failure outpatients at high risk of hospital readmission. J Am Coll Cardiol. 2002;39:471-480.

10. GARÍN O, SORIANO N, RIBERA A, FERRER M, PONT A, ALONSO J, et al. Validation of the Spanish version of the Minnesota Living with Heart Failure Questionnaire. Rev Esp Cardiol 2008;61:251-259.

11. DITEWIG JB, BLOK H, HAVERS J, VAN VEENENDAAL $H$. Effectiveness of self-management interventions on mortality, hospital readmissions, chronic heart failure hospitalization rate and quality of life in patients with chronic heart failure: A systematic review. Patient Educ Couns $2010 ; 78: 297-315$.

12. GOTT M, BARNES S, PARKER C, PAYNE S, SEAMARK D, GARIBALLA $S$, et al. Predictors of the quality of life of older people with heart failure recruited from primary care. Age and Ageing. 2006; 35: 172 -177.

13. CLARK DO, TU W, WEINER M, MURRAY MD. Correlates of health-related quality of life among lower-income, urban adults with congestive heart failure. Heart \& Lung: The Journal of Acute and Critical Care. 2003; 32:391-401.

14. HEO S, MOSER DK, RIEGEL B, HALL LA, CHRISTMAN N. Testing a Published Model of Health-Related Quality of Life in Heart Failure. Journal of Cardiac Failure. 2005;11:372-379.

15. MCALISTER FA, STEWART S, FERRUA S, MCMU-
RRAY JJ. Multidisciplinary strategies for the management of heart failure patients at high risk for admission: A systematic review of randomized trials. Journal of the American College of Cardiology. 2004;44:810-819.

16. HARRISON M, BROWNE G, ROBERTS J, TUGWELL P, GAFNI A. Quality of Life of Individuals With Heart Failure: A Randomized Trial of the Effectiveness of Two Models of Hospital-to-Home Transition. Medical care. 2002;40:271-286

17. HOLLAND R, BATTERSBY J, HARVEY I, LENAGHAN E, SMITH J, HAY L. Systematic review of multidisciplinary interventions in heart failure. Heart. 2005;91:899 -906.

18. RAMACHANDRAN K, HUSAIN N, MAIKHURI R, SETH S, VIJ A, KUMAR M, et al. Impact of a comprehensive telephone-based disease management programme on quality of life in patients with heart failure. Nat Med $\mathrm{J}$ India 2007;20:67-73.

19. DEL SINCADO D, PULIGNANO G, MINARDI G, APOSTOLI A, GUERRIERI L, ROTOLONIM, et al. Twoyear outcome of a prospective, controlled study of a diseasemanagement programme for elderly patients with heart failure. J Cardiovasc Med (Hagers-town) 2007; 8: 324-9.

20. ATIENZA F, ANGUITA M, MARTINEZ-ALZAMORAN, OSCA J, OJEDA S, ALMENAR L, et al. Multicenter randomized trial of a comprehensive hospital discharge and outpatient heart failure management program. Eur J Heart Fail 2004;6:643-52.

21. BRUGGINK-ANDRE`DE LA PORTE PW, LOK DJ, VAN VELDHUISEN DJ, VANWIJNGAARDEN J, CORNEL JH, ZUITHOFF NP, et al. Added value of a physician-and nurse directed heart failure clinic: results from the Deventer-Alkmaar heart failure study.Heart 2007; 93: 819-25.

22. DUNAGANWC, LITTENBERG B, EWALD GA, JONES CA, EMERY VB, WATERMAN BM, et al. Randomized trial of a nurse-administered, telephone-based disease management program for patients with heart failure. J Card Fail 2005;11:358-65

23. SHIVELY M, KODIATH M, SMITH TL, KELLY A, 
BONE P, FETTERLY L, et al. Effect of behavioral management on quality of life in mild heart failure: a randomized controlled trial. Patient Educ Couns 2005;58:27-34.

24. MARTENSSON J, STRO "MBERG A, DAHLSTRO "MU, KARLSSON JE, FRIDLUND B. Patients with heart failure in primary health care: effects of a nurse-led intervention onhealth-related quality of life and depression. Eur J Heart Fail 2005;7:393-403.

25. WIERZCHOWIECKI M, POPRAWSKI K, NOWICKA A, KANDZIORA M, PIATKOWSKA A, JANKOWIAK $\mathrm{M}$, et al. A new programme of multidisciplinary care for patients with heart failure in Poznan: one-year follow-up. Kardiol Pol 2006;64:1063-70.

26. BOCCHI EA, CRUZ F, GUIMARAES G, PINHO MOREIRA LF, ISSA VS, AYUB FERREIRA SM, et al. Longterm prospective, randomized, controlled study using repetitive education at six-month intervals and monitoring for adherence in heart failure outpatients: the REMADHE trial. Circ Heart Fail 2008;1:115-124.

27. DELANEY C, APOSTOLIDIS B. Pilot testing of a multicomponent home care intervention for older adults with heart failure: an academic clinical partnership. J Cardiovasc Nurs 2010;25:E27-40.

28. BLUE L, LANG E, MCMURRAY JJV, DAVIE AP, MCDONAGH TA, MURDOCH DR, et al. Randomised controlled trial of specialist nurse intervention in heart failure. BMJ. 2001;323:715-718.

29. FERRANTE D, VARINI S, MACCHIA A, SOIFER $S$, BADRA R, NUL D, et al. Long-term results after a telephone intervention in chronic heart failure: DIAL (Randomized Trial of Phone Intervention in Chronic Heart Failure) follow-up. J Am Coll Cardiol 2010;56:372-378.

30. CLARKE M, SHAH A, SHARMA U. Systematic review of studies on telemonitoring of patients with congestive heart failure: a meta-analysis. J Telemed Telecare 2011;17:7-14.

31. CAPOMOLLA S, FEBO O, CERESA M, CAPOROTONDI A, GUAZZOTTI G, LA ROVERE MT, et al. Cost/utility ratio in chronic heart failure: comparison between heart failure management program delivered by day-hospital and usual care. J Am Coll Cardiol. 2002;40:1259-1266.

32. GARY RA, DUNBAR SB, HIGGINS MK, MUSSELMAN DL, SMITH AL. Combined exercise and cognitive behavioral therapy improves outcomes in patients with heart failure. J Psychosom Res 2010;69:119-131.

33. ANDERSEN K, JONSDOTTIR S, SIGURETHSSON AF, SIGURETHSSON SB. The effect of physical training in chronic heart failure. Laeknabladid 2006;92:759-764.

34. MICHE E, ROELLEKE E, ZOLLER B, WIRTZ U, SCHNEIDER M, HUERST $M$, et al. A longitudinal study of quality of life in patients with chronic heart failure following an exercise training program. Eur J Cardiovasc Nurs 2009;8:281-287.

35. VAN TOL BA, HUIJSMANS RJ, KROON DW, SCHOTHORST M, KWAKKEL G. Effects of exercise training on cardiac performance, exercise capacity and quality of life in patients with heart failure: a meta-analysis. Eur J Heart Fail 2006;8:841-850.

36. PINA IL. Exercise and heart failure: A statement from the American Heart Association Committee on exercise, rehabilitation, and prevention. Circulation 2003;107:1210-1225.

37. WANG TJ, EVANS JC, BENJAMIN EJ, LEVY D, LEROY EC, VASAN RS. Natural History of Asymptomatic Left Ventricular Systolic Dysfunction in the Community. Circulation. 2003;108:977-982.

38. RODRIGUEZ-ARTALEJO F, GUALLAR-CASTILLON P, PASCUAL CR, OTERO CM, MONTES AO, GARCIA AN, et al. Health-related quality of life as a predictor of hospital readmission and death among patients with heart failure. Arch Intern Med 2005;165:1274-1279.

39. STEWART S. Financial aspects of heart failure programs of care. European Journal of Heart Failure. 2005;7:423 -428.

40. INGLIS SC, PEARSON S, TREEN S, GALLASCH T, HOROWITZ JD, STEWART S. Extending the Horizon in Chronic Heart Failure: Effects of Multidisciplinary, HomeBased Intervention Relative to Usual Care. Circulation. 2006;114:2466-2473. 